\title{
Effects of the IncRNA ENST00000623984 on colon cancer and the biological characteristics of colon cancer cells
}

\author{
Zhi-bao Liu, ${ }^{1,2}$ Jing-hua Zhang, ${ }^{2}$ Jing-hua Gao, ${ }^{2}$ Jian Shi ${ }^{1}$ \\ ${ }^{1}$ Oncology Department, The Fourth Hospital of Hebei Medical University, Shijiazhuang, Hebei \\ ${ }^{2}$ Oncology Department, Cangzhou Central Hospital, Cangzhou, Hebei, China
}

\begin{abstract}
The aim of this study was to explore the effects of the lncRNA ENST00000623984 on colorectal cancer. In this study, the expression levels of ENST000000623984 were first examined in tumor tissue and adjacent normal tissue from 40 patients with colorectal cancer and LoVo cells using quantitative real-time PCR. By siRNA transfection, ENST00000623984 expression was knocked down. Using flow cytometry, cell cycle progression and cell viability were examined in basal and knockdown LoVo cells. The CCK-8 assay was used to assess the cell proliferation rate, and the transwell assay was used to determine the migration and invasion abilities. The ENST000000623984 expression level was increased in colorectal cancer. Knockdown of ENST000000623984 reduced cell viability, proliferation rate, cell migration and invasion. These results suggested that lncRNA ENST000000623984 may be involved in colorectal cancer development.
\end{abstract}

Key words: LncRNA; ENST00000623984; colorectal cancer; expression; PCR.

Correspondence: Jian Shi, Oncology Department, The Fourth Hospital of Hebei Medical University, No. 12 Health Road, Shijiazhuang, 050011, Hebei, China. E-mail: qgj663@163.com

Contributions: ZBL, JS, carried out the studies, participated in collecting the data, analyzed the data, drafted the manuscript; JHZ, JHG, performed the statistical analysis and participated in the study design. All authors have read and approved the final manuscript.

Availability of data and materials: The data and materials in this study are available from the corresponding author on reasonable request.

Ethics approval: Ethical approval was obtained for all experimental procedures by the Ethical Committee of the Fourth Hospital of Hebei Medical University.

Patient consent for publication: Written informed consent was obtained from the patients for their anonymized information to be published in this article.

Conflict of interest: The authors declare no competing interests.

Funding: This study received no funding 


\section{Introduction}

Colorectal cancer is a common gastrointestinal malignancy. The disease is the third most prevalent cancer and the second most common cause of cancer death worldwide with an annual death rate of more than 608,700 according to the National Cancer Institute. ${ }^{1}$

Long noncoding RNAs (lncRNAs), with a length of more than 200 nucleotides, have been shown to play regulatory roles in eukaryotes, serving as, for instance, antisense, intergenic transcript and epigenetic regulators, which may be involved in cell proliferation, migration, apoptosis and tumor development. ${ }^{2-4}$ lncRNAs have been found to be deregulated in multigenetic diseases, including Alzheimer's disease, heart disease and cancer. ${ }^{5-10}$ Some lncRNAs can act as tumor suppressors, such as MEG $3^{11}$ and CPS11T1, in lung cancer and hepatocellular carcinoma, ${ }^{12,13}$ while some lncRNAs may act as tumor promotors, such as MALAT1, which enhances metastasis and cell proliferation in esophageal squamous carcinoma and glioma. ${ }^{14,15}$ Despite the accumulating studies of lncRNAs in colorectal cancer, ${ }^{16-19}$ many other lncRNAs remains poorly defined and characterized.

Our previous finding of a whole transcriptome resequencing study from 3 patients with colorectal cancer revealed 91 lncRNAs that showed different expression patterns between the tumor tissue and the adjacent normal tissue (unpublished), indicating that these lncRNAs may be involved in the development of colorectal cancer. Among these lncRNAs, ENST00000623984, which showed obvious differences in the whole transcriptome resequencing study, was selected to further explore its role in colorectal cancer, as well as in LoVo colorectal cancer cells, in the current study.

\section{Materials and Methods}

\section{Clinical tissues}

The study was approved by the Institutional Ethics Committee of the Fourth Hospital of Hebei Medical University, and written informed consent was obtained from all participants. Forty (40) patients diagnosed with colorectal cancer were recruited for this study. The patients had not been treated with radiotherapy or chemoradiotherapy. Tumor and adjacent normal tissues were collected from each patient, snap-frozen in liquid nitrogen and stored at $-80^{\circ} \mathrm{C}$ for subsequent analysis.

\section{Immunohistochemistry}

Some of the tissues were removed from the cancerous tissue and adjacent tissues of each colorectal cancer patient and fixed with $4 \%$ paraformaldehyde for $48 \mathrm{~h}$. After fixation, paraffin embedding was performed, and the embedded wax block was sliced. The slices were deparaffinized in water, placed into antigen retrieval solution, placed at low heat for $10 \mathrm{~min}$, and cooled to room temperature. The slides were washed in PBS and incubated in $3 \% \mathrm{H}_{2} \mathrm{O}_{2}$ for $15 \mathrm{~min}$ at room temperature to eliminate endogenous peroxidase activity. Then, Ki-67 (1:500 dilution; WL03319, Wanleibio, Shenyang, China) or MMP9 (1:500 dilution; WL03096, Wanleibio, Shenyang, China) primary antibody, diluted with PBS, was added to evenly cover the tissue and incubated overnight at $4^{\circ} \mathrm{C}$. Then, HRP-goat anti-rabbit IgG secondary antibody diluted with PBS (1:2000 dilution; 111-035-003, Jackson ImmunoResearch, West Grove, PA, USA) was added and incubated for $1 \mathrm{~h}$ at room temperature. Finally, DAB color development, hematoxylin counterstaining, dehydrating and transparent reagents, and mounting medium were added. Images of the slides were captured using a microscope. Image-Pro Plus software was used to analyze the optical density of the photos.

\section{Cell culture and transfection}

The human colorectal cancer cell line LoVo was purchased from the American Type Culture Collection in China (Shanghai, China) and routinely maintained in DMEM supplemented with $10 \% \mathrm{FBS}$ and $2 \mathrm{mM} \mathrm{L-glutamine} \mathrm{at} 37^{\circ} \mathrm{C}$ with $5 \% \mathrm{CO}_{2}$, followed by a passage once every 2-3 days until cells reaches the logarithmic growth phase. Small-interfering RNA (siRNA) targeting lncRNA ENST00000623984 and negative control plasmids were purchased from Invitrogen (Carlsbad, CA, USA) (Table 1). Lipofectamine 2000 (Invitrogen) was used as the vector in cell transfection according to the manufacturer's instructions. At $24 \mathrm{~h}$ after transfection, the transfection efficiency was assessed under a fluorescence microscope to determine whether subsequent experiments could be performed. The cells were collected at appropriate time points for further experiments based on the experimental requirements.

\section{Cell death and cell cycle assays}

When the cells reached $90 \%$ confluence, they were trypsinized, washed, resuspended, and seeded in a 6-well plate overnight. Then, they were transfected and recovered for $24 \mathrm{~h}$. The cells were harvested and washed twice with PBS and resuspended in $1 \mathrm{x}$ binding buffer. Then, $5 \mu \mathrm{L}$ annexin V-FITC and $5 \mu \mathrm{L}$ propidium iodide (PI, $1 \mu \mathrm{g} / \mathrm{mL}$ ) were added to $1 \times 10^{5}$ cells. The cells were incubated in the dark for $15 \mathrm{~min}$ at room temperature. Then, $400 \mu \mathrm{L}$ of $1 \mathrm{x}$ binding buffer was added. The fraction of dead cells was assessed at this stage via NovoCyte (Aceabio, Santa Clara, CA, USA). Additionally, the cells were further subjected to a cell cycle test following the instructions of the Cell Cycle Kit (WLA010a, Wanleibio, Shenyang, China). Generally, the cells were washed twice with PBS, trypsinized and washed once in PBS. Approximately $1 \times 10^{6}$ cells were incubated in $70 \% \mathrm{EtOH}$ at $4^{\circ} \mathrm{C}$ overnight, followed by a washing in PBS twice. Approximately $100 \mu \mathrm{L}$ of RNAse A $(0.25 \mathrm{mg} / \mathrm{mL})$ was used to resuspend the cells, and they were incubated at $37^{\circ} \mathrm{C}$ for $30 \mathrm{~min}$. The cells were then treated with $500 \mu \mathrm{L}$ PI $(50 \mu \mathrm{g} / \mathrm{mL})($ WLA001a, Wanleibio, Shenyang, China) and incubated at $4^{\circ} \mathrm{C}$ in the dark for $30 \mathrm{~min}$. At least $1 \times 10^{4}$ cells for each sample were measured with an excitation wavelength of $488 \mathrm{~nm}$ and the emission wavelength at $530 \mathrm{~nm}$ for FITC and $620 \mathrm{~nm}$ for PI, respectively. The percentage of cell cycle phases was quantified using FlowJo software (FlowJo LLC, Ashland, OR, USA).

\section{RT-qPCR}

Total RNA from each well of cells or tissue from a patient was isolated by using TRIzol (15596026, Invitrogen), followed by $0.2 \mathrm{~mL}$ of chloroform (Sigma-Aldrich, St. Louis, MO, USA). After centrifugation, the RNA was pelleted by mixing with $0.5 \mathrm{~mL}$ of isopropyl alcohol and centrifugation. The resulting pellet was washed with $700 \mu \mathrm{L}$ of $75 \%$ ethanol and finally resuspended in RNAse-free water (Ambion, USA). The RNA was stored at $-80^{\circ} \mathrm{C}$

Table 1. SiRNA sequences used for knocking down lncRNA ENST00000623984.

\begin{tabular}{ll} 
Item & \multicolumn{1}{c}{ Sequence } \\
IncRNA ENST00000623984 siRNA & 5'-CCUGGCUUCCAGGGCAUAATT-3' \\
& 5'-UUAUGCCCUGGAAGCCAGGTT-3' \\
Negative control & 5'-UUCUCCGAACGUGUCACGUTT-3' \\
& 5'-ACGUGACACGUUCGGAGAATT-3' \\
\hline
\end{tabular}


until use

cDNA was synthesized from 500 ng RNA using a reverse transcription reagent kit (Life Technology, Carlsbad, CA, USA). For real time PCR, $0.25 \mu \mathrm{M}$ of each primer in $10 \mu \mathrm{L}$ was used (Table $2)$. $\beta$-Actin was used as the housekeeping gene. The relative expression levels of genes were calculated using the comparative delta $\mathrm{Cq}\left(2^{-\Delta \Delta \mathrm{Ct}}\right)$ method. ${ }^{20}$.

\section{Cell viability}

After overnight seeding, cells were washed with $500 \mu \mathrm{L}$ PBS (Shuangluoxuan Biological Technology Co., Ltd., Jiangsu, China). The transfection mixture was then added to the wells. Each treatment was performed in quintuplicates. The cells were cultured at $37^{\circ} \mathrm{C}$ with $5 \% \mathrm{CO}_{2}$ for $24 \mathrm{~h}, 48 \mathrm{~h}$ or $72 \mathrm{~h}$. After incubation, $10 \mu \mathrm{L}$ of CCK-8 (WLA074a; Wanleibi) was added to each well. The cell viability was then determined using an ELISA microplate reader (BIOTEK) at $450 \mathrm{~nm}$.

\section{Invasion/migration assay}

Transwell assays were performed at $24 \mathrm{~h}$ after transfection. Cells were transferred to the upper chamer with 3000 cells in $0.1 \mathrm{~mL}$ serum-free medium per well. Matrigel (Millipore, MA, USA)-coated membranes were used in invasion assay, and uncoated membranes were used to carry out migration assay. In both assays, the lower transwell chamber was filled with medium supplemented with $20 \%$ FBS. Cells were cultured for $2 \mathrm{~h}$. After that, membrane was collected, cleaned and subjected to $0.5 \%$ crystal violet (Sigma-Aldrich) staining at room temperature for $14 \mathrm{~min}$. Cells were evaluated under an optical microscope (Olympus, Tokyo, Japan).

\section{Statistics}

The SPSS 17.0 (SPSS, Inc., Chicago, IL, USA) statistical software was used for statistical analysis in this study. Results were expressed with the mean \pm standard deviation (SD) from 3 biological replicates. The $t$-test was used for the comparison of continuous variables between two groups, and ANOVA was used for the comparison of continuous variables among groups; $\mathrm{p}<0.05$ was considered statistically significant.

\section{Results}

\section{IncRNA ENST00000623984 expression is higher in tumor tissue}

To examine the role of ENST00000623984 in colorectal cancer, the expression levels of ENST00000623984 were detected in tumor tissue and adjacent normal tissue from 40 patients with colorectal cancer using real-time PCR. The relative expression level in the tumor tissue was $0.001 \pm 0.000056$, and that in normal tissue was $0.0004 \pm 0.000025$ (Figure 1). The difference was statistically significant $(\mathrm{p}<0.001)$.

Expression levels of anti- Ki67 and anti-MMP9 proteins

\section{in patient tissues}

To determine the expression levels of Ki-67 and MMP9 in patient tissues, immunohistochemical methods were used to detect the expression of these two proteins in 40 pairs of cancer tissues and adjacent tissues. The average optical density of Ki-67 and MMP9 in cancer tissues was 1.4 times and 4.9 times that in adjacent tissues, respectively, and the differences were statistically significant (Figure $2 \mathrm{~A}, \mathrm{~B})$.

\section{The expression of ENST00000623984 in LoVo cells}

To further explore the role of lncRNA ENST00000623984, the colorectal cancer cell line LoVo was used as a model, and the expression levels were examined in these cells. The results showed that ENST00000623984 was expressed in LoVo cells, and the corresponding siRNA was able to reduce ENST00000623984 expression levels from $1.00 \pm 0.06$-fold to $0.23 \pm 0.02$-fold (Figure 3 ). The expression level in the negative control cells was $1.08 \pm 0.04$-fold (Figure 3).

\section{The effects of ENST00000623984 on cell cycle}

In LoVo cells, 24h after transfection, ENST00000623984 knockdown caused more cells to arrest at $\mathrm{G}_{1}$ phase $(82.13 \%)$ and fewer cells to arrest at $S$ phase $(7.17 \%)$ and $G_{2}$ phase $(10.7 \%)$ than in the basal group $\left(\mathrm{G}_{1}=66.24 \%, \mathrm{~S}=12.77 \%, \mathrm{G}_{2}=20.99 \%\right)$ and negative control group $\left(\mathrm{G}_{1}=67.52 \%, \mathrm{~S}=10.92 \%, \mathrm{G}_{2}=21.56 \%\right)$ (Figure 4 A,4B). Thus, cells were less likely to replicate when ENST00000623984 was knocked down. Therefore, ENST00000623984 may promote LoVo cell cycle progression.

\section{The effect of ENST00000623984 on the cell prolifera- tion rate}

At $24 \mathrm{~h}$, the OD values shows no significant difference between ENST00000623984 knockdown cells and the basal cells

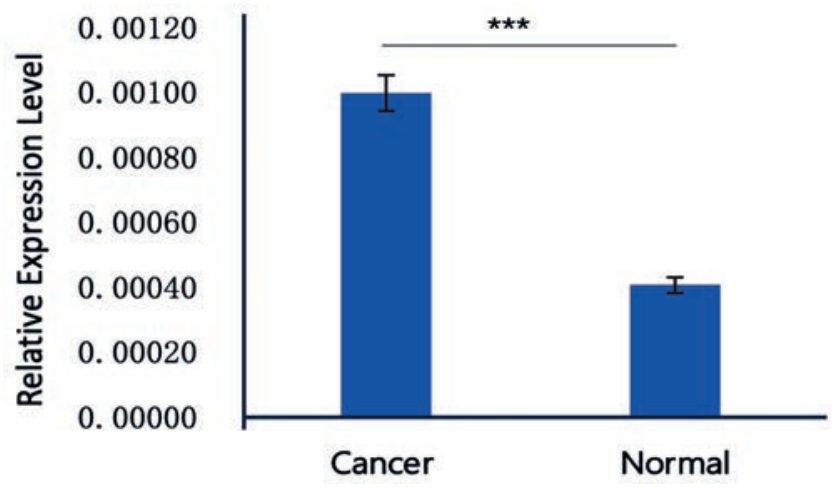

Figure 1. The expression level of lncRNA ENST00000623984 in colorectal tumor tissue was significantly higher than that in adjacent normal tissue. (mean $\pm \mathrm{SD}, \mathrm{n}=40$; $\left.{ }^{* * *} \mathrm{p}<0.001\right)$.

Table 2. Primers used in real-time PCR.

\begin{tabular}{lcccc} 
Primer & Sequence & Primer length & Product sive \\
IncRNA F & CAGGACTAGACTTAGGAGGAG & 21 & 50.2 & $174 \mathrm{bp}$ \\
IncRNA R & GATATGGAATCGGTGGT & 18 & 49.4 & $155 \mathrm{bp}$ \\
$\beta$-actin F & CACTGTGCCCATCTACGAGG & 20 & 58.6 & 56.8 \\
$\beta$-actin R & TAATGTCACGCACGATTTCC & 20 & 5 & \\
\hline
\end{tabular}


as well as the negative control cells. While comparing to the basal cells and negative control cells, the OD values in ENST00000623984 knockdown cell was significantly lower at $48 \mathrm{~h}$ and $72 \mathrm{~h}(\mathrm{p}<0.05)$ (Figure 5). As the OD values were positively correlated with the cell proliferation rate, indicating that the proliferation rate of LoVo cells could be inhibited by ENST00000623984 knockdown.

\section{The effect of ENST00000623984 on cell apoptosis}

The average cell apoptosis rate of the ENST00000623984 knockdown cells $(13.87 \pm 0.2)$ was significantly higher than that of the basal cells $(2.79 \pm 0.1)$ and the negative control cells $(2.93 \pm 0.1)$ $(\mathrm{p}<0.001)$ (Figure 6). This results demonstrates that knockdown of ENST00000623984 may promote the apoptosis of LoVo cells.

\section{The effect of ENST00000623984 on cell invasion}

The number of LoVo cells invading the Matrigel-coated microporous membrane in ENST00000623984 knockdown cells (45 \pm 5$)$ was much lower than that in basal cells $(70 \pm 7)$ and negative control cells (66 \pm 7 ) (Figure 7). Therefore, knockdown of lncRNA ENST0000023984 led to inhibited invasion of LoVo cells.

\section{The effect of ENST00000623984 on cell migration}

The number of migratory LoVo cells was $97 \pm 10$ in the basal group, $97 \pm 11$ in the negative control group, and $72 \pm 7$ in the
A
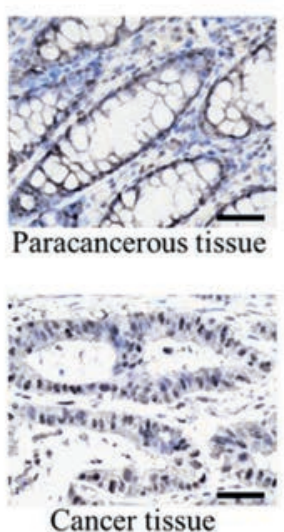

Cancer tissue

B
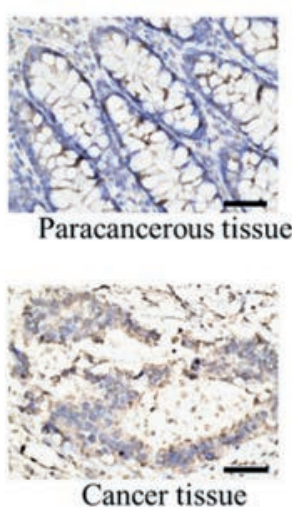
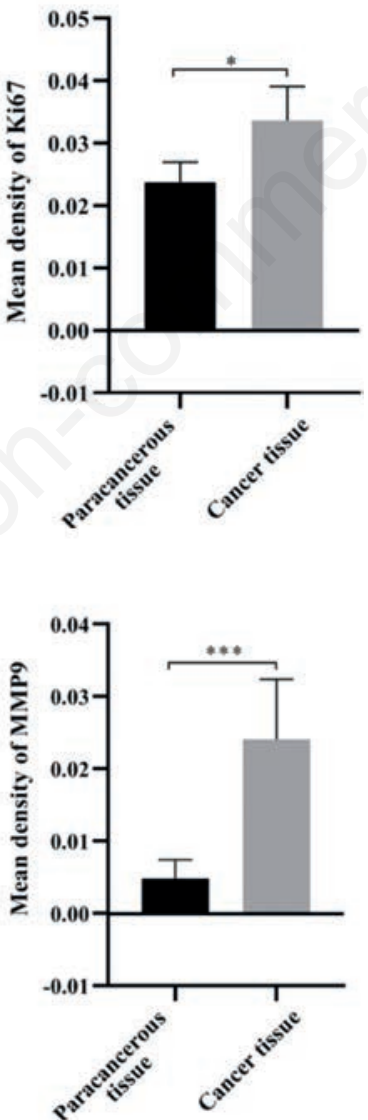

Figure 2. The expression of Ki-67 and MMP9 in adjacent and cancerous tissues. A) Ki-67 expression level. B) MMP9 expression level. ${ }^{*} \mathbf{p}<0.05 ;{ }^{* * *} \mathbf{p}<0.001$.

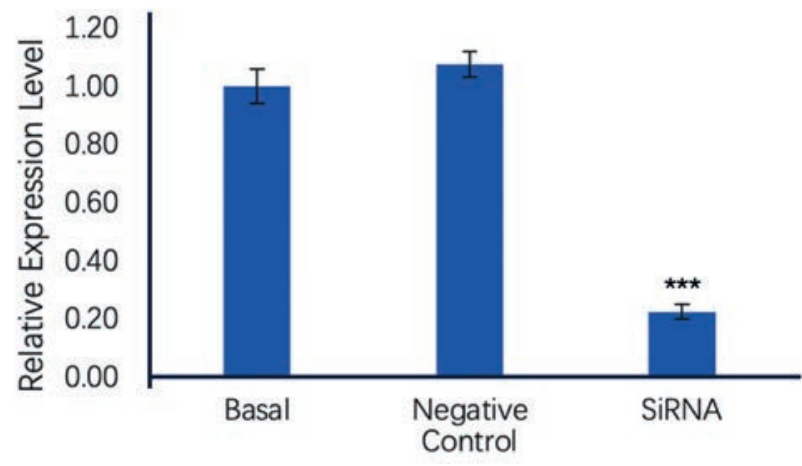

Figure 3. The expression of lncRNA ENST00000623984 in LoVo cells. The expression level of IncRNA ENST00000623984 in was significantly lower in cells transfected with ENST00000623984 siRNA than that in basal cells and negative control cells. ${ }^{* * *} \mathbf{p}<0.001$.

ENST00000623984 knockdown group (Figure 8), suggesting that ENST00000623984 tended to promote LoVo cell migration.

A
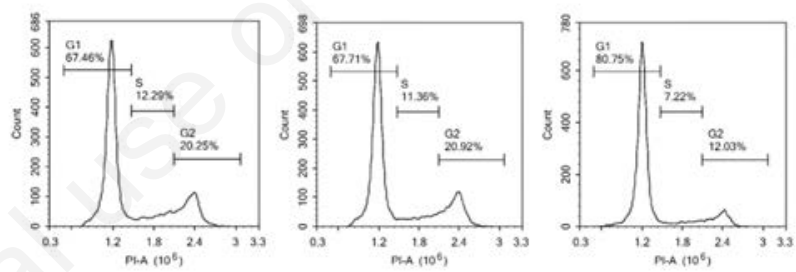

B

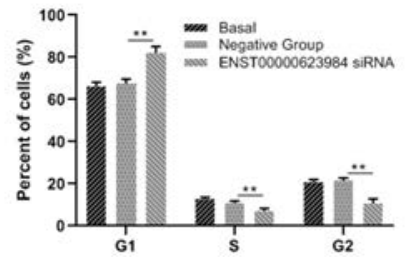

Figure 4. The effect of ENST00000623984 knockdown on cell cycle progression. A) Flow cytometry was used to test the cell cycle in basal LoVo cells (left), negative control cells (middle), and ENST00000623984 knockdown cells (right). B) ENST00000623984 knockdown could inhibit cell cycle progression. ${ }^{* *} \mathrm{p}<\mathbf{0 . 0 1}$.

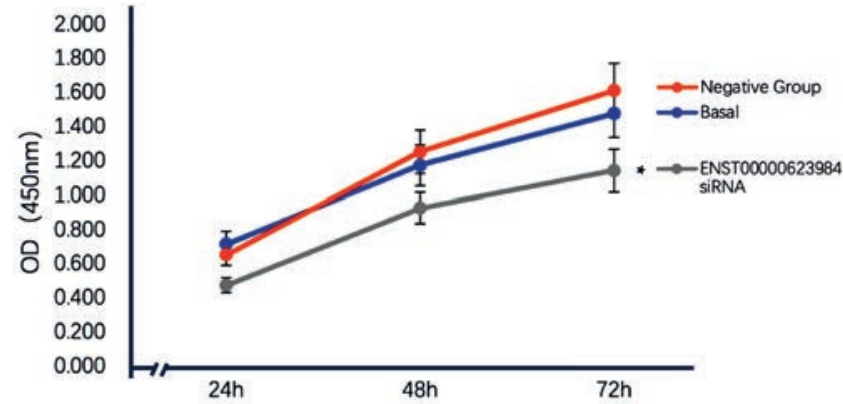

Figure 5. Knockdown of ENST00000623984 reduced cell viability. Growth curves were determined via the CCK8 assay for LoVo cells which were untransfected (blue line), transfected with negative control (orange line), or transfected with ENST00000623984 siRNA (gray line) respectively. ${ }^{*} \mathrm{p}<0.05$. 
A

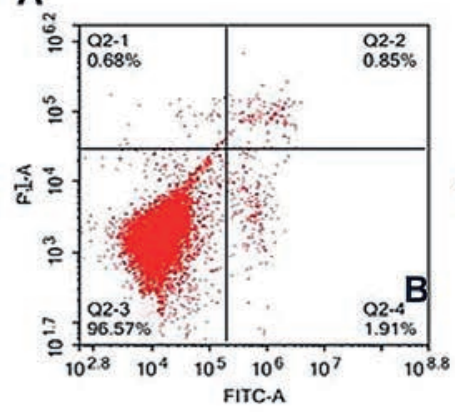

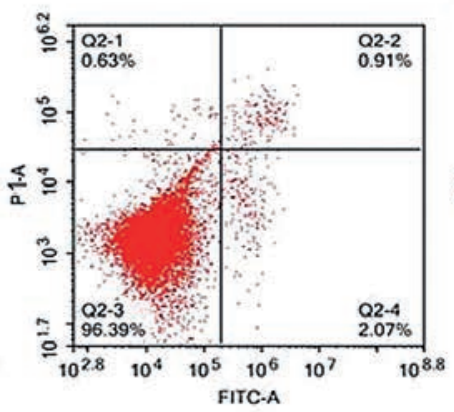

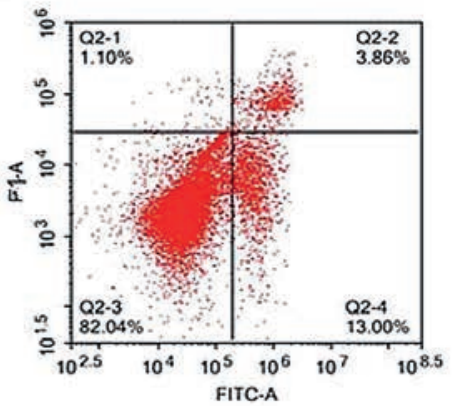

B

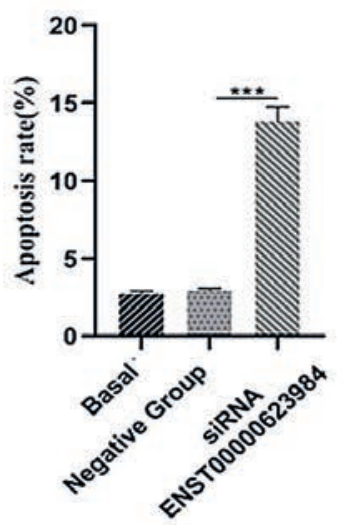

Figure 6. Knockdown of ENST00000623984 promoted LoVo cell apoptosis. A) Flow cytometry was used to test the apoptotic rates in normal LoVo cells (left), in cells transfected with negative shRNA (middle), and in cells transfected with ENST00000623984 siRNA (right). B) Knockdown of ENST00000623984 promoted apoptosis of LoVo cells. ${ }^{* * *} \mathrm{p}<0.001$.

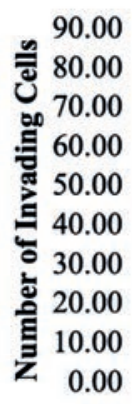

Figure 7. Knockdown of ENST00000623984 reduced the number of invading LoVo cells. Comparing to the basal cells and negative control cells, the number of invading LoVo cells reduced significantly in ENST00000623984 siRNA-transfected cells. ${ }^{* * *} \mathbf{p}<0.001$.

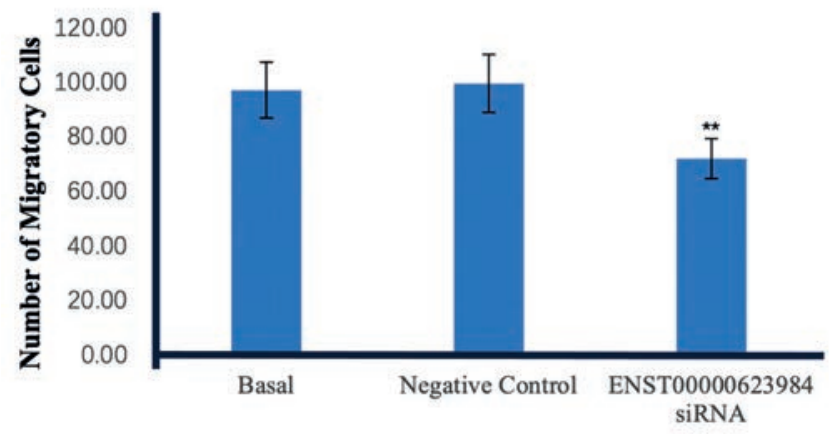

Figure 8. Knockdown of ENST00000623984 reduced the number of migratory LoVo cells. The number of migratory cells in ENST00000623984 siRNA-transfected group was significantly lower than that in basal cells, negative control cells. ${ }^{* *} \mathrm{p}<0.01$.

\section{Discussion}

lncRNA ENST00000623984 was revealed in a whole transcriptome resequencing study, where it was upregulated by 58 -fold in tumor tissue compared to adjacent normal tissue (unpublished). In the current study, a similar expression pattern was detected in the tumor tissue from 40 patients with colorectal cancer using RTPCR. These results indicate a potential role of ENST00000623984 in colorectal cancer development. To further explore the role of ENST00000623984, the colorectal cancer cell line LoVo was used. ENST00000623984 was expressed in LoVo cells, and siRNA could knock down its expression in these cells. ENST00000623984 knockdown inhibited the proliferation of LoVo cells and reduced cell viability. This evidence suggests that ENST00000623984 may be a potential stimulator in colorectal tumor development.

Metastases are the major cause of cancer-related death, and migration and invasion are strongly associated with metastases. In this study, ENST00000623984 enhanced the invasion and migration of LoVo cells, so it might positively regulate colorectal cancer metastases. The different expression patterns of ENST00000623984 between tumor tissue and normal tissue indicate that this lncRNA could serve as a prognostic biomarker for colorectal cancer. Dysregulation of lncRNAs is involved in cellular growth advantage that may lead to progressive and uncontrolled tumor growth. ${ }^{21-25}$ Knockdown of lncRNA ENST00000623984 showed an anticancer effect, in which cell viability, cell proliferation rate, and invasion and migration abilities were decreased. Anti-ENST00000623984 might be a potential treatment for colorectal cancer. The mechanism behind ENST00000623984 positively regulating tumor development needs to be further explored.

\section{References}

1. Torre LA, Bray F, Siegel RL, Ferlay J, Loetet-Tieulent J, Jemal A. Global cancer statistics. CA Cancer J Clin 2011;61:69-90.

2. Cech TR, Steitz JA. The noncoding RNA revolution-trashing old rules to forge new ones. Cell 2014;157:77-94.

3. Ling H, Fabbri M, Calin GA. MicroRNAs and other non-cod- 
ing RNAs as targets for anticancer drug development. Nat Rev Drug Discov 2013;12:847-65.

4. Wang TH, Lin YS, Chen Y, Yeh CT, Huang YL, Hsieh TH, et al. Long non-coding RNA AOC4P suppresses hepatocellular carcinoma metastasis by enhancing vimentin degradation and inhibiting epithelial-mesenchymal transition. Oncotarget 2015;6:23342-57.

5. Liu Y, Pan S, Liu L, Zhai X, Liu J, Wen J, et al. A genetic variant in long non-coding RNA HULC contributes to risk of HBV-related hepatocellular carcinoma in a Chinese population. PLoS One 2012; 7:e35145.

6. Schonrock N, Harvey RP, Mattick JS. Long noncoding RNAs in cardiac development and pathophysiology. Circ Res 2012;111:1349-62.

7. Batista P, Chang H. Long noncoding RNAs: Cellular address codes in development and disease. Cell 2013;152:1298-307.

8. Faghihi MA, Modarresi F, Khalil AM, Wood DE, Sahagan BG, Morgan TE, et al. Expression of a noncoding RNA is elevated in Alzheimer's disease and drives rapid feed-forward regulation of beta-secretase. Nat Med 2008;14:723-30.

9. Li X, Wu Z, Fu X, Han W. Long noncoding RNAs: Insights from biological features and functions to diseases. Med Res Rev 2013;33:517-53.

10. Tan L, Yu JT, Hu N, Tan L. Non-coding RNAs in Alzheimer's disease. Mol Neurobiol 2013;47:382-93.

11. Zhang X, Zhou Y, Mehta KR, Danila DC, Scolavino S, Johnson SR, et al. A pituitary-derived MEG3 isoform functions as a growth suppressor in tumor cells. J Clin Endocrinol Metab 2003;88:5119-26.

12. Wang TH, Yu CC, Lin YS, Chen TC, Yeh CT, Liang KH, et al. Long noncoding RNA CPS1-IT1 suppresses the metastasis of hepatocellular carcinoma by regulating HIF-1 $\alpha$ activity and inhibiting epithelial-mesenchymal transition. Oncotarget 2016;7:43588-603.

13. Zhao XG, Liu MR, Zhang JJ, Zhang RS, Zhang Q, Tan XF. Long noncoding RNA CPS1-IT1 suppresses cell proliferation and metastasis in human lung cancer. Oncol Res 2017;25:37380.

14. Hu L, Wu Y, Tan D, Meng H, Wang K, Bai Y, et al. Up-regu- lation of long noncoding RNA MALAT1 contributes to proliferation and metastasis in esophageal squamous cell carcinoma. J Exp Clin Cancer Res 2015;34:7.

15. Ma KX, Wang HJ, Li XR, Li T, Su G, Yang P, et al. Long noncoding RNA MALAT1 associates with the malignant status and poor prognosis in glioma. Tumour Biol 2015;36:3355-9.

16. Silva-Fisher JM, Dang HX, White NM, Strand MS, Krasnick BA, Rozycki EB, et al. Long non-coding RNA RAMS11 promotes metastatic colerectal cancr progression. Nat Commun 2020;11:2156.

17. Liu TY, Han ZY, Li HY, ZhuYK, Sun ZQ, Zhu AL. LncRNA DLEU1 contributes to colorectal cancer progression via activation of KPNA3. Mol Cancer 2018;17:118.

18. Tang R, Chen JH, Tang MT, Liao ZQ, Zhou LQ, Jiang JR, et al. LncRNA SLCO4A1-AS1 predicts poor prognosis and promotes proliferation and metastasis via the EGFR/MAPK pathway in coloretal cancer. Int J Biol Sci 2019;15:2885-96.

19. Chen S, Liu Y, Wang YY, Xue ZP. LncRNA CCAT1 promotes colorectal cancer tumorigenesis via miR-181b-5p/TUSC3 axis. Onco Targets Ther 2019;12:9215-25.

20. Livak KJ and Schmittgen TD. Analysis of relative gene expression data using real-time quantitative PCR and the 2 (-Delta Delta C(T)) method. Methods 2001;25:402-8.

21. Prensner JR, Chinnaiyan AM. The emergence of lncRNAs in cancer biology. Cancer Discov 2011;1:391-407.

22. Liao Z, Nie H, Wang Y, Luo J, Zhou J, Ou C. The emerging landscape of long non-coding RNAs in colorectal cancer metastasis. Front Oncol 2021;11:641343.

23. Zhang J, Li K, Zheng H, Zhu Y. Research progress review on long non-coding RNA in colorectal cancer. Neoplasma 2021:68:240-52.

24. Talebi A, Azizpour M, Agah S, Masoodi M, Mobini GR, Akbari A. The relevance of long noncoding RNAs in colorectal cancer biology and clinical settings. J Cancer Res Ther 2020;16:S22-33.

25. Schwarzmueller L, Bril O, Vermeulen L, Léveillé N. Emerging role and therapeutic potential of lncRNAs in colorectal cancer. Cancers (Basel) 2020;12:3843.

Received for publication: 30 December 2020. Accepted for publication: 22 May 2021.

This work is licensed under a Creative Commons Attribution-NonCommercial 4.0 International License (CC BY-NC 4.0).

(C) Copyright: the Author(s), 2021

Licensee PAGEPress, Italy

European Journal of Histochemistry 2021; 65:3215

doi:10.4081/ejh.2021.3215 